(Aus dem thierphysiol. Laboratorium der kgl. landwirthsch. Hochschule zu Berlın.)

\title{
Einfluss der Geschwindigkeit, der Körpertemperatur und der Uebung auf den Stoffverbrauch bei Ruhe und bei Muskelarbeit.
}

Von

N. Zuntz.

Die Versuche, welche Herr Slowtzoff in vorstehender Abhandlung zum Studium des Einflusses der Körpergrösse auf den Verbrauch beim Gehen benutzt hat, erscheinen insofern nicht alle streng vergleichbar, als die Geschwindigkeit der verschiedenen Thiere eine verschieden grosse war. Sie wurde von Herrn Slowtz off nach dem Behagen der Thiere regulirt. Es fragt sich, ob die Resultate durch die Unterschiede der Gehgeschwindigkeit erheblich beeinflusst sind. Beim Pferde hatte ich mit Hagemann ${ }^{1}$ ) einen sehr bedeutenden Einfluss der Gesehwindigkeit gefunden. Für jedes Meter Zunahme der Minutengeschwindigkeit wuchs der Energieaufwand für die horizontale Fortbewegung des Körpers um 1,03\% der bei $78 \mathrm{~m}$ Geschwindigkeit erforderlichen Energie. Beim Menschen fand Dr. Leo Zuntz und ich selbst mit Schumburg ${ }^{2}$ ) den Effect geringer. Der Mehraufwand bei rascherem Gehen betrug zwischen 60 und $100 \mathrm{~m}$ bei drei geubten Menschen:

$$
\left.\begin{array}{l}
0,84 \% \\
0,42 \% \\
0,39 \%
\end{array}\right\} \begin{gathered}
\text { pro Meter Zuwachs der } \\
\text { Geschwindigkeit }
\end{gathered}
$$

Bei starker Verlangsamung des Ganges unter das übliche Maass nimmt sogar der Verbrauch für die Wegeeinheit wieder zu gegenüber den mittleren Werthen, wie ich mit Schumburg (1. c. S. 293) aus den Angaben von Frentzel und Reach berechnet habe. Aus

1) Zuntz und Hagemann, Untersuchungen uber den Stoffwechsel des Pferdes S. $309 \mathrm{ff}$.

2) Schumburg und Zuntz, Physiologie des Marsches S. 284-288. Berlin 1901. 
Einfluss der Geschwindigkeit, der Korpertemperatur u. der Uebung etc. 193

dem Gesagten geht hervor, dass es nicht möglich ist, aus den vorliegenden Versuchen am Menschen und Pferde die Bedeutung des verschieden schnellen Ganges fur den Stoffwechsel des Hundes abzuschätzen.

Ich hatte schon bei meinen ersten Versuchen über den Stoffverbrauch des arbeitenden Hundes (dieses Arch. Bd. 68 S. 198 Tab. IV) auf den Einfluss der Geschwindigkeit auf den Stoffbedarf für die Horizontalbewegung geachtet. Es schien damals, als ob dieser Einfluss geringer sei als beim Pferde, vielleicht ganz fehle.

Ich bin jetzt in der Lage, noch einige Daten zur Beurtheilung dieser Frage zu geben.

Der zu meinen zahlreichen Versuchen mit Dr. W. Loeb ${ }^{1}$ ) (jetzt Privatdocent in Bonn) benutzte, vorzuglich eingeubte Hund ging zwei Mal mit einer Geschwindigkeit von ca. $100 \mathrm{~m}$, wobei er traben musste, während er vorher und nachher in flottem Schritt 55 bis $58 \mathrm{~m}$ machte. Die Versuchsdaten sind folgende:

Tabelle I.

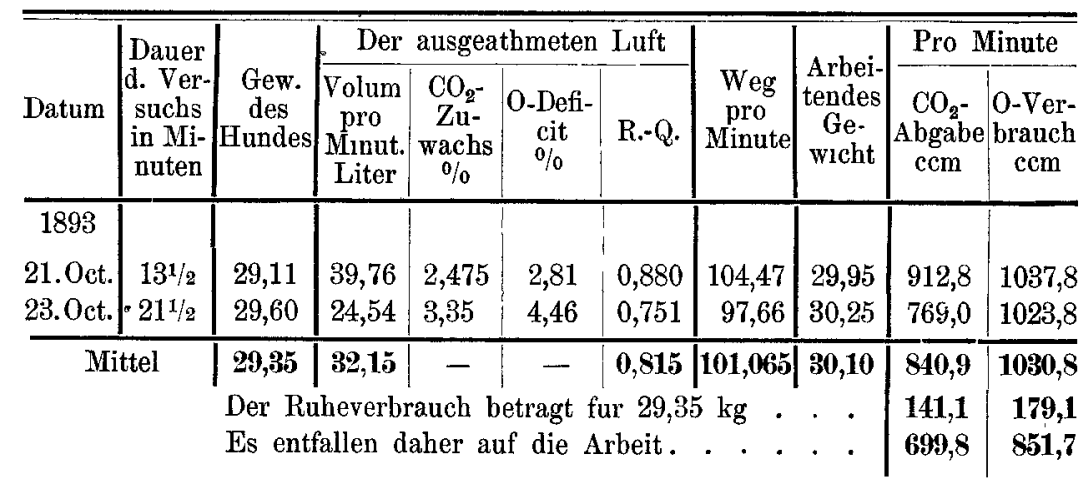

Die Arbeit bestand in Bewegung von $30,1 \mathrm{~kg}$ uber $101,065 \mathrm{~m}$. Es bedingt daher die Bewegung von $1 \mathrm{~kg}$ uber $1000 \mathrm{~m}$ einen Sauerstoffverbrauch von $\frac{851,7 \times 1000}{30,1 \times 101,065} 279,98=\mathrm{ccm}$.

Die Bahn hatte eine Steigung von $0,16 \%$, es wurde also bei Bewegung von $1 \mathrm{~kg}$ uber $1000 \mathrm{~m}$ noch $1,6 \mathrm{mkg}$ Steigarbeit geleistet, welche bei diesem Hunde $1,6 \times 1,173=1,877 \mathrm{ccm}$ Sauerstoff erfordert, so dass $278,10 \mathrm{ccm}$ Sauerstoff für die reine Horizontalbewegung nöthig waren. Der resp. Quot. war hierbei $\frac{699,8}{851,7}=0,822$,

1) Verhandl. d. physiol. Gesellsch, zu Berlin, 3. Aug. 1894. 
wobei $1 \mathrm{ccm}$ Sauerstoff $=4,828 \mathrm{cal}$. Die verbrauchten $278,1 \mathrm{ccm}$ Sauerstoff entsprechen daher 1342 cal. An denselben zwei Tagen betrug der Kraftverbrauch im Mittel der sechs von Herrn Slowtz off in Tab. 19 zusammengestellten Versuche 1343 cal. bei $57,4 \mathrm{~m}$ Minutenweg. Der Verbrauch ist also durch die grosse Zunahme der Geschwindigkeit gar nicht beeinflusst.

Ein einziger ähnlicher Versuch liegt an der von Herrn Slow$\mathrm{t} z$ off mit Nr. 5 bezeichneten Hündin vor. Folgendes sind die bezüglichen Daten.

Tabelle II.

\begin{tabular}{|c|c|c|c|c|c|c|c|c|c|c|}
\hline \multirow[b]{2}{*}{ Datum } & \multirow{2}{*}{$\begin{array}{l}\text { Dauer } \\
\text { d. Ver- } \\
\text { suchs } \\
\text { in Mi- } \\
\text { nuten }\end{array}$} & \multirow[b]{2}{*}{$\begin{array}{c}\text { Grew. } \\
\text { des } \\
\text { Hundes }\end{array}$} & \multicolumn{4}{|c|}{ Der ausgeathmeten Luft } & \multirow[b]{2}{*}{$\begin{array}{c}\text { Weg } \\
\text { pro } \\
\text { Minute }\end{array}$} & \multirow[b]{2}{*}{$\begin{array}{l}\text { Arbei- } \\
\text { tendes } \\
\text { Ge- } \\
\text { wicht }\end{array}$} & \multicolumn{2}{|c|}{ Pro Minute } \\
\hline & & & $\begin{array}{l}\text { Volum } \\
\text { pro } \\
\text { Minut. }\end{array}$ & $\mid \begin{array}{c}\mathrm{CO}_{2^{-}} \\
\mathrm{Zu}- \\
\text { wachs } \\
\% / 0\end{array}$ & 0-Defi- & R.-Q. & & & $\begin{array}{c}\mathrm{CO}_{2} \\
\text { Abgabe }\end{array}$ & $\begin{array}{l}\text { O-Ver- } \\
\text { brauch }\end{array}$ \\
\hline 1893 & & & & & & & & & & \\
\hline 11.Nov. & 14 & 28,41 & 16,69 & 3,575 & 4,705 & 0,760 & 99,98 & 24,25 & 585,4 & 770,4 \\
\hline & & $\begin{array}{l}\text { Ruhev } \\
\text { Gaswe }\end{array}$ & chsel & die & . & $\cdot$ & - & . & $\begin{array}{r}99,8 \\
485,6\end{array}$ & $\begin{array}{l}139,3 \\
631,1\end{array}$ \\
\hline
\end{tabular}

Aus diesen Daten berechnet sich unter Berücksichtigung einer Steigung von $0,03 \%$ für $1 \mathrm{~kg}$ und $1000 \mathrm{~m}$ Weg: bei rund $100 \mathrm{~m}$ per Minute: 259,7 cem O-Verbrauch und 1237 cal. -

Dagegen war das Mittel von 5 Versuchen im Schritt bei $58,6 \mathrm{~m}$ per Minute: $235,3 \mathrm{ccm}$ O-Verbrauch und $1123 \mathrm{cal}$.

Für einen Zuwachs der Geschwindigkeit um 41,4 m wächst der Kraftverbrauch um 114 cal., d. h. für $1 \mathrm{~m}$ Geschwindigkeitszuwachs um 2,75 cal. oder um $0,24 \%$ des Verbrauchs bei $58,6 \mathrm{~m}$ Geschwindigkeit. Hier ist die Steigerung zwar vorhanden, aber doch geringer, als sie in einem der Versuche am Menschen gefunden wurde.

Bei derselben Hundin finden sich unter unseren Versuchen mit stärkerem Bergaufsteigen, aus welchen Herr Slowtzoff seine Tabelle 26 zusammengestellt hat, noch 5 dort nicht verwerthete (Datum 5. October 1893), bei welchen der Minutenweg nur 41,48 m beträgt. Man kann sie mit den Werthen vom 13. October und 17. October mit durchschnittlich $56,8 \mathrm{~m}$ Geschwindigkeit vergleichen. Ich stelle sie zu diesem Behufe in Tabelle III zusammen: 
Einfluss der Geschwindigkeit, der Körpertemperatur u. der Uebung etc. 195

Tabelle III.

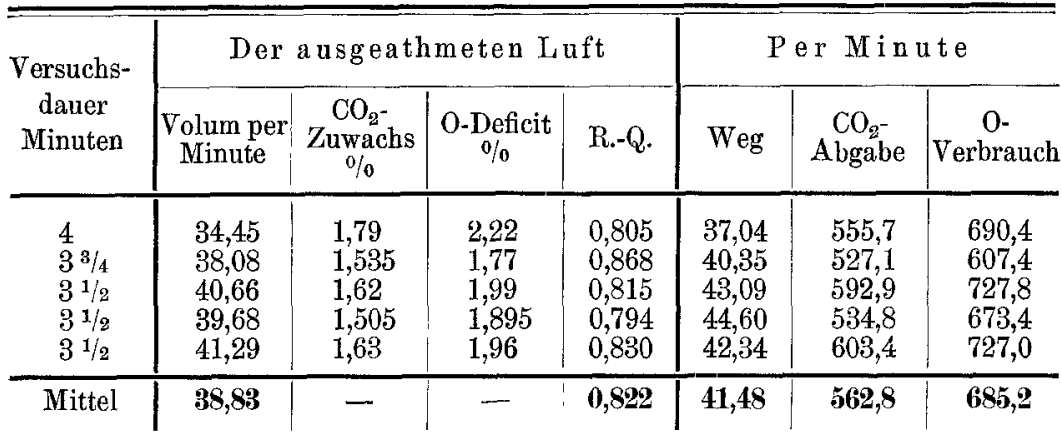

Das Lebendgewicht betrug zur Zeit dieses Versuches $23,55 \mathrm{~kg}$, demnach der Gaswechsel in Ruhe: $100,3 \mathrm{ccm} \mathrm{CO} 2140,1 \mathrm{ccm} O$. Die Steigerung durch die Arbeit betrug: $462,5 \mathrm{~cm} \mathrm{CO}_{2} 545,1 \mathrm{ccm} 0$. Das Thier wog mit Geschirr u. s. w. $24,45 \mathrm{~kg}$. Es wurden daher pro Kilogramm und $1000 \mathrm{~m}$ Weg verbraucht $\frac{545,1 \times 1000}{24,45 \times 41,48}=$ 537,45 cem O. Das entspricht bei R.-Q. $=0,848$. . $2612 \mathrm{cal}$.

Beim Vergleich mit Herrn Slowtz off's Tabelle 26 sehen wir, dass der Verbrauch beim langsamen Gang hier grösser ist als beim schnelleren. Für die Bewegung von $1 \mathrm{~kg}$ úber $1000 \mathrm{~m}$ unter gleichzeitiger Hebung um $142 \mathrm{~m}$ braucht die Hundin:

5 Versuche vom 5 . October bei $41,5 \mathrm{~m}$ Geschwindigkeit $537,4 \mathrm{ccm} 0$ und 2612 cal.

6 Versuche vom 13 . October bei $57,5 \mathrm{~m}$ Geschwindigkeit $482,7 \mathrm{ccm} 0$ und 2261 cal.

5 Versuche vom 17 . October bei $57,0 \mathrm{~m}$ Geschwindigkeit $471,4 \mathrm{~cm} \mathrm{C}$ und 2297 cal.

Es ist nicht daran zu denken, dass der Minutenweg von $41,5 \mathrm{~m}$ dem Hunde wegen der Langsamkeit des Gehens unbequem ist; die auf den ersten Blick paradoxe Erscheinung erklärt sich vielmehr aus der

\section{Steigerung der Körperwärme,}

welche die Hündin in diesen Versuichen erlitten hat. Dieselbe documentirt sich auch in der von Versuch zu Versuch wachsenden Athemgrösse, welche im ersten Versuche 28 Liter per Minute, im letzten 41,3 Liter bei gleicher Arbeitsleistung betrug. Dafur, dass bei erheblicherem Wachsen der Temperatur der Stoffverbrauch des 
arbeitenden Hundes bei unveränderter Arbeit wächst, habe ich noch eine Anzahl Belege gefunden. Ich möchte hier nur eine am 11. October ausgeführte Versuchsreihe an derselben Hündin vorlegen, bei welcher die Körpertemperatur nach Beendigung der $1 \% 3$ Stunden anhaltenden Steigarbeit $41,7^{\circ}$ C. betrug; dementsprechend stieg die Athemgrösse während der letzten Arbeitsperiode bis auf 72 Liter, und der Kohlensäuregehalt betrug nur $1,39 \%$, - also eine ganz charakteristische thermische Polypnoè.

Die wichtigsten Daten dieser interessanten Versuchsreihe stelle ich in Tabelle IV zusammen':

Tabelle IV.

\begin{tabular}{|c|c|c|c|c|c|c|c|}
\hline \multirow{2}{*}{$\begin{array}{l}\text { Versuchs- } \\
\text { dauer } \\
\text { Minuten }\end{array}$} & \multicolumn{4}{|c|}{ In der ausgeathmeten Luft } & \multicolumn{3}{|c|}{ Per Minute } \\
\hline & $\begin{array}{c}\text { Athem- } \\
\text { grosse per } \\
\text { Minute }\end{array}$ & $\begin{array}{c}\text { Kohlen- } \\
\text { saure- } \\
\text { zuwachs }\end{array}$ & $\begin{array}{l}\text { Sauer- } \\
\text { stoff- } \\
\text { deficit }\end{array}$ & $\begin{array}{l}\text { Resp. } \\
\text { Quot. }\end{array}$ & $\begin{array}{l}\text { Sauerstoff- } \\
\text { aufnahme } \\
\text { ccm }\end{array}$ & $\begin{array}{c}\text { Kohlen- } \\
\text { saure-Aus- } \\
\text { scherdung } \\
\text { cem }\end{array}$ & $\underset{\mathrm{m}}{\text { Weg }}$ \\
\hline $\begin{array}{l}9 \\
13 \\
10 \\
91 / 3 \\
8^{3 / 4} \\
8^{3}\end{array}$ & $\begin{array}{l}45,16 \\
51,48 \\
55,43 \\
58,86 \\
66,17 \\
72,05\end{array}$ & $\begin{array}{l}1,755 \\
1,57 \\
1,445 \\
1,275 \\
1,245 \\
1,36\end{array}$ & $\begin{array}{l}1,885 \\
2,00 \\
1,59 \\
1,65 \\
1,72 \\
1,60\end{array}$ & $\begin{array}{l}0,931 \\
0,785 \\
0,909 \\
0,773 \\
0,725 \\
0,851\end{array}$ & $\begin{array}{r}772,22 \\
932,95 \\
795,96 \\
875,72 \\
1021,07 \\
1030,26\end{array}$ & $\begin{array}{l}717,97 \\
732,37 \\
723,37 \\
676,70 \\
740,38 \\
876,28\end{array}$ & $\begin{array}{l}59,976 \\
57,195 \\
59,005 \\
57,330 \\
58,211 \\
57,550\end{array}$ \\
\hline Mittel & 58,191 & 1,4416 & 1,7408 & 0,829 & 904,70 & 744,51 & 58,211 \\
\hline
\end{tabular}

Die Bahn hatte wie in den anderen Versuchen an diesem Thiere eine Steigung von $14,2 \%$. Das Thier wog mit Geschirr und Trachealcanüle $24,2 \mathrm{~kg}$. Auf $1 \mathrm{~kg}$ bewegtes Gewicht und $1000 \mathrm{~m}$ berechnet sich hier $543,3 \mathrm{ccm}$ Sauerstoffverbrauch $2637 \mathrm{cal}, \quad$ also ein noch etwas höherer Verbrauch als in den Versuchen vom 5. October. Hier ist aber auch die Lungenventilation noch um 20 Liter höher als dort. So mag der gesteigerte Verbrauch zum Theil sicher auf die grössere Arbeit der Athemmuskeln zuruckzufuhren sein. Wenn unsere Erfahrungen am Menschen und am Pferde auch auf den Hund übertragen werden durfen, würde die Verstärkung der Ventilation um 20 Liter den Sauerstoffverbrauch um 60-100 ccm in die Höhe treiben. -

Dass aber die erhöhte Körperwärme an sich die Oxydation steigert, ist ja fủr den ruhenden Organismus mehrfach, am präcisesten durch die zahlreichen Versuche von Pfläger (dieses Arch. Bd. 18 S. 305, 321, 356) dargethan worden. 
In Pfl u̇g e 's Versuchen bedingte Steigerung der Körperwärme über die Norm eine Erhöbung des Sauerstoffverbrauchs pro Grad um $10 \%$ bei curaresirten Thieren,

" $6,1 \%$ "durch hohe Rückenmarksdurchschneidung gelähmten Thieren,

" 5,7\% " unverletzten Thieren.

Bei unserer Hundin haben wir es, da das Thier nach den anderen Arbeitsversuchen nur 39,6 bis $39,9^{\circ}$ C. maass, mit einer Ueberwärmung von etwa $2^{\circ} \mathrm{C}$. zu thun.

Hierfür können wir eine Steigerung der Oxydation des ruhenden Thieres um $15 \%=21 \mathrm{ccm} \mathrm{O}$ annehmen.

Fur 20 Liter Mehrathmung $20 \times 4=80 \mathrm{ccm} 0$. Im Ganzen $101 \mathrm{ccm}$.

Ziehen wir diese Zahl von dem zu 904,7 ccm gefundenen Sauerstoffverbrauch während der Arbeit ab, so bleiben $803,7 \mathrm{ccm}$, woraus sich in der gewohnten Weise der Aufwand pro Kilogramm und $1000 \mathrm{~m}$ Weg berechnet zu:

$$
471,6 \mathrm{cem} 0 \text { und } 2289 \mathrm{cal} \text {. }
$$

Der so corrigirte Werth ist mit den bei Vermeidung der Ueberhitzung gefundenen ubereinstimmend.

Wir hatten nämlich

am 13. October 482,7 cem 0 und 2261 cal.

"17. " $471,4 \mathrm{ccm} \mathrm{O} \mathrm{,2297"}$

Es erscheint demnach gerechtfertigt, die Ursache des grösseren Energieverbrauchs der überhitzten Thiere bei gleicher Arbeit in der gesteigerten Athemleistung und in dem von der Arbeit unabhängigen erhöhten Zerfall zu suchen.

Die als Kraftquelle dienende Steigerung des Umsatzes im arbeitenden Muskel selbst bleibt dieselbe, wenigstens für eine Körperwärme bis zu $41,7^{\circ} \mathrm{C}$. - Dass in diesem und einigen anderen Versuchen bei einer Arbeit, welche durchaus nicht übermässig war, und bei gủnstiger Lufttemperatur (hier $17^{\circ} \mathrm{C}$.) so gewaltige Steigerungen der Körpertemperatur eintraten, hat seinen Grund in der Störung der Wärmeregulation, welche die Tracheotomie herbeiführt. Da die Haut des Hundes nur sehr mangelhaft mit Schweissiruisen ausgerüstet ist, kommt wesentlich die Verdunstung von der Lungenoberfläche und von der mit Speichel befeuchteten Zunge als Regulationsmittel in Betracht, wo die Strahlung und Leitung nicht genügt. Die Verdunstung von der Lunge erfolgt natürlich nach der Tracheotomie 
bei gleicher Menge und Beschaffenheit der Athemluft ebenso wie sonst, mit dem einzigen Unterschiede, dass der allerdings nur kleine Theil der inspirirten Luft, welcher die Trachealcanule und die kurze Schlauchleitung füllt, keinen Wasserdampf aufnimmt, während bei normaler Athmung die Dampfsättigung sich zu einem grossen Theil schon in der Nase vollzieht. Wine einfache Ueberschlagsrechnung lehrt aber, dass selbst bei Nasenathmung die Wassermenge, welche auf diese Weise bei excessivster Steigerung der Lungenventilation verdampft, bei Weitem nicht ausreicht, um die in den thätigen Muskeln gebildete Wärme abzufubren ${ }^{x}$ ).

Eine' grössere Wassermenge verdunstet von der mit Speichel úberrieselten Zungenoberfläche, indem der uber sie hingeblasene Strom der Exspirationsluft von den Seiten noch eine mehrfach grössere Menge der relativ trockenen Aussenluft ansaugt und dadurch viel Wasser zur Verdunstung bringt. Man kann sich von der Bedeutung dieser der Wirkung einer Strahlpumpe analogen Luftzufuhr über die feuchte Zunge leicht uberzeugen, wenn man mit dem Munde uber den Handrucken bläst, einmal während derselbe trocken, dann wenn er befeuchtet ist. Im ersteren Falle erzeugt die warme und mit Wasserdampf gesättigte Athemluft ein Gefühl von Wärme, im letzteren eine sehr ausgesprochene Kalteempfindung. Die Verdunstungskälte kann, da die direct aus dem Munde kommende Luft mit Wasserdampf gesättigt ist, nur durch aspirirte Aussenluft zu Stande kommen. Diese Förderung der Verdunstung von der Zunge durch die stossweise, hachelnd darüber hingeblasene Luft fehlt nun dem tracheotomirten Hunde, und das ist der Grund, wesshalb er sich nicht ge-

1) Die Rechnung wurde in der dieses Archiv Bd 92 S. 487 dargelegten Weise ausgefuhrt; sie ergab, dass be1 58 Liter Minutenventilation wahrend der Arbeitszeit von 104 Minuten sicher nicht $300 \mathrm{~g}$ Wasserdampf in die Athemluft übergehen konnien. Der Weg betrug $5800 \mathrm{~m}$; da pro Kilogramm und $1000 \mathrm{~m}$ 2,6 Cal. gebracht wurden, bedingt dies eine Warmeproduction von 5,800 $\times 24,2 \times 2,6$ $=365$ Cal.; davon werden 46 Cal. zur Hebung des Korpers benutzt; 319 Cal. sind als Warme fortzuschaffen. Dazu mússen, wenn keine vermehrte Strahlung zu Hulfe kommt, $\frac{319}{0,54}=591 \mathrm{~g}$ Wasser verdampfen, also doppelt so viel, als die Athmung leisten konnte. Normal ist aber die Lungenventilation und damit auch die Verdampfung durch die Lunge nur etwa halb so gross; es konnen von der Lunge nur $150 \mathrm{~g}$ Wasser verdampfen, wahrend die Zungenoberflache uber $400 \mathrm{~g}$ zu hefern hat; eine Zahl, de durch die gesteigerte Strahlung von der Hant wohl nur wenig vermindert wird. 
nügend entwärmen kann. An einem warmen Sommertage ist mir ein tracheotomirter Hund, welcher etwas uber 1 Stunde die gewohnte Arbeit des Bergaufsteigens auf der Tretbahn geleistet hatte, plötzlich todt zusammengebrochen. Fin bald nachher in den After geschobenes Thermometer zeigte $45^{\circ} \mathrm{C}$.

Nach diesen Erfahrungen haben wir bei allen länger dauernden, messend verwertheten Arbeitsversuchen an tracheotomirten Hunden die Haut in kurzen Zwischenraumen mit reinem Wasser oder solchem, welches $15-20 \%$ Alkohol enthielt, benetzt. Dadurch konnte man das Steigen der Körperwärme verhuten. Die Häufigkeit der Waschungen wurde nach der Athmung des Hundes bestimmt; sobald dieselbe starker hachelnd und oberflächlich zu werden anfing, fand eine Benetzung statt.

\section{Der Einfluss der Uebung}

auf Verminderung des Stoffverbranches bei verschiedenartigen Leistungen ist vielfach messend erwiesen worden. In den Versuchen von Hagemann und mir an Pferden, von Schumburg und mir bei marschirenden Soldaten finden sich zahlreiche Belege dafur. Gruber und Schnyder baben unter Kronecker's Leitung die Stoffersparniss bei vorschreitender Uebung studirt. Bei Erörterung der Versuche von Newton Heineman (dieses Archiv Bd. 83 S. $561 \mathrm{ff}$.) konnte ich darthun, dass bei seinem Versuchsindividuum der Stoffverbrauch für dieselbe, durch Raddrehen geleistete Arbeit von Monat zu Monat geringer wurde; dass also hier Monate vergingen, ehe das Optimum der Arbeitsleisung erreicht war. Im Gegensatz hierzu war bei den marschierenden Soldaten dies Ziel schon nach 4-5 Uebungsmarschen erreicht. So wird es uns nicht Wunder nehmen, wenn auch bei den von Dr. Slowtzoff untersuchten Hunden Unterschiede in der Erlangung der vollen Uebung sich zeigten. Eine ungefähre Vorstellung darùber, wie weit etwa Aenderungen im Verbrauch durch die fortschreitende Uebung bedingt sind, gewinnt man bei Betrachtung des letzten Stabes der Tabellen: Sauerstoffverbrauch pro Kilo und $1000 \mathrm{~m}$ Weg. Diese Zahlen sind zwar nicht streng unter sich vergleichbar, weil der Ruheverbrauch nicht abgezogen ist und daher eine Zahl zu gross im Vergleich mit dem Durchschnitt wird, wenn der zuruekgelegte Weg unter dem durchschnittlichen blieb und umgekehrt. Mit Berücksichtigung dieses Umstandes erkennt man, dass in den meisten Tabellen die Zahlen 
regellos um den Mittelwerth schwanken. Hier war offenbar die stets längere Zeit vor Beginn der messenden Versuche ausgeführte Trainirung der Hunde ausreichend gewesen, um dieselben zu möglichst ökonomischem Gehen zu bringen. In einigen Tabellen aber zeigen die ersten Versuche die höchsten Werthe; hier war also durch die Vorübung noch nicht die vollkommenste Ausnutzung der Muskelkraft erreicht worden.

Ein eigenthümliches Verhalten zeigt Hund Nr. 6. Derselbe war ein gut dressirter Ziehhund, gewohnt, weite Wege in den ebenen Strassen Berlins am Wagen zurückzulegen. Ich benutzte ihn nach dem Ankauf sofort zu den Versuchen, welche ich in diesem Arehiv Bd. 68 S. 191 beschrieben habe. Zwei Jahre später diente er Dr. Loeb und mir zu den Versuchen, welche von Dr. Slowtz off in Tabelle XIX und XXVII zusammengefasst sind. In dieser Zeit ging er vom 19. September bis zum 1. November täglich einen Weg von $6,35 \mathrm{~km}$ bei $14,2 \%$ Steigung, nur an $\mathrm{z}$ wei T'age $\mathrm{n}$, am 21. und 23. October, ging er dieselbe Strecke horizontal. (Tabelle XIX.) Bei diesem Horizontalweg brauchte er pro Kilogramm und $1000 \mathrm{~m}$ 1343 cal., während er 2 Jahre vorher nur 1179 cal. gebraucht hatte. Dagegen betrug der Verbrauch für $1 \mathrm{mkg}$ Steigarbeit damals 7,668 cal., und 1893 bei der stetigen Uebung im Bergaufsteigen nur 5,867 cal. - Dieser sehr niedrige Werth fur die Steigarbeit kommt natúrlich dadurch zu Stande, dass der Abzug fúr die Horizontalcomponente des Ganges hier so hoch gefunden wurde. Aber selbst wenn wir mit dem 1891 gefundenen niedrigen Verbrauch rechnen, finden wir fur die Steigarbeit einen geringeren Verbrauch als damals, nämlich 7,02 cal. fur $1 \mathrm{mkg}$. -

Wir sehen hier, dass der Hund jedes Mal die Arbeit, auf welche er gerade speciell eingeubt war, am ökonomischsten leistete; 1893 war dies die Steigarbeit, 1891 der horizontale Gang. -

Sehr deutlich tritt noch eine fortschreitende Sauerstoffersparniss bei der Arbeit in den Versuchen an Hund 7 bei schwacher Steigung hervor. Hier ist desshalb ein zu hoher Werth des Verbrauchs für die Horizontalbewegung gefunden worden. Wenn wir die letzten 4 Versuche der Tabelle XX allein berücksichtigen, finden wir als Mittel pro Minute bei $37,11 \mathrm{~m}$ Weg, $36,2 \mathrm{~kg}$ Lebendgewicht und $36,93 \mathrm{~kg}$ arbeitendes Gewicht:

$$
490,35 \mathrm{cem} \mathrm{CO}_{2} \quad 591,22 \mathrm{cem} \mathrm{O}
$$


Einfluss der Geschwindigkeit, der Kórpertemperatur u. der Uebung etc. 201

Hiervon ist der Ruheverbrauch abzuziehen, welcher aber, wie S. 169 ausgefúhrt, im Mittel der 9 Versuche von Tabelle VII zu hoch gefunden wurde, weil der Hund meist mit Muskelspannung lag. Nur in den nach stärkerer Arbeit ausgeführten Versuchen lag der Hund mit entspannten Muskeln. Diese 2 Versuche ergeben pro Kilogramm und Minute im Mittel

$3,845 \mathrm{cem} \mathrm{CO} \quad 5,23 \mathrm{cem} \mathrm{O}$.

Das macht auf $36,2 \mathrm{~kg}$ Lebendgewicht $139,19 \mathrm{cem} \mathrm{CO} \quad 189,33 \mathrm{cem} \mathrm{O}$.

Also Arbeitsverbrauch: $351,16 \mathrm{ccm} \mathrm{CO}_{2} 401,89 \mathrm{ccm} \mathrm{O}$.

Unter Anwendung derselben Ruhewerthe wird der Arbeitsverbrauch beim Bergaufsteigen (23,6\% Steigung):

$$
687,0 \mathrm{cem} \mathrm{CO} 2989,3 \mathrm{ccm} \mathrm{O}
$$

Die Seite 189 aufgestellten Gleichungen lauten auf Grund dieser Zahlen :

1. $x+236,0 y=604,6 \mathrm{~cm} 0=2823 \mathrm{cal}$.

2. $x+42,9 y=293,2 \mathrm{cem} 0=1435$ cal.

Hieraus: $x=224,1 \mathrm{cem} 0=1126 \mathrm{cal} .=479 \mathrm{mkg}=1,583 \mathrm{mkg}$ pro

Oberflächeneinheit.

Hieraus: $y=1,612 \mathrm{cem} 0=7,192 \mathrm{cal} .=3,056 \mathrm{mkg}$.

Durch diese Correctur wird eine Unregelmässigkeit in der von Herrn Slowtzoff aufgestellten Reihe beseitigt. Der grösste der Hunde steht jetzt mit 1126 cal. an der unteren Grenze des Verbrauchs für die Horizontalbewegung, und der nahe Parallelismus dieses Verbrauchs zu $P^{2 / 3}$ oder mit anderen Worten zum Quadrat der Körperlänge tritt noch deutlicher hervor. Statt der von Herrn Slowtz off eingestellten Zahl $1,757 \mathrm{mkg}$ fur $K^{2 / 3}$ ergibt sich jetzt als richtige Zahl $1,583 \mathrm{mkg}$.

Zur leichteren Beurtheilung des ganzen Thatsachenmaterials stelle ich in Tabelle $\mathrm{V}$ alle berichtigten Werthe noch einmal zusammen und gebe zugleich für jede Zahl die Abweichung von dem Mittel aller untersuchten Hunde. Um den Ruheverbrauch bequemer mit den sonst ublichen Zahlen vergleichen zu können, berechne ich denselben auf 24 Stunden.

Beim Anblick der Tabelle fällt sofort auf, dass der Verbrauch für die Steigarbeit bei den einzelnen Thieren zwar recht erheblich von dem Mittelwerth $3,00 \mathrm{mkg}$ ehemischer Energie fur $1 \mathrm{mkg}$ Steigarbeit abweicht, dass aber diese Abweichungen in keiner Beziehung zur Körpergrösse stehen, wie Herr Slowtz off schon betont hat. 


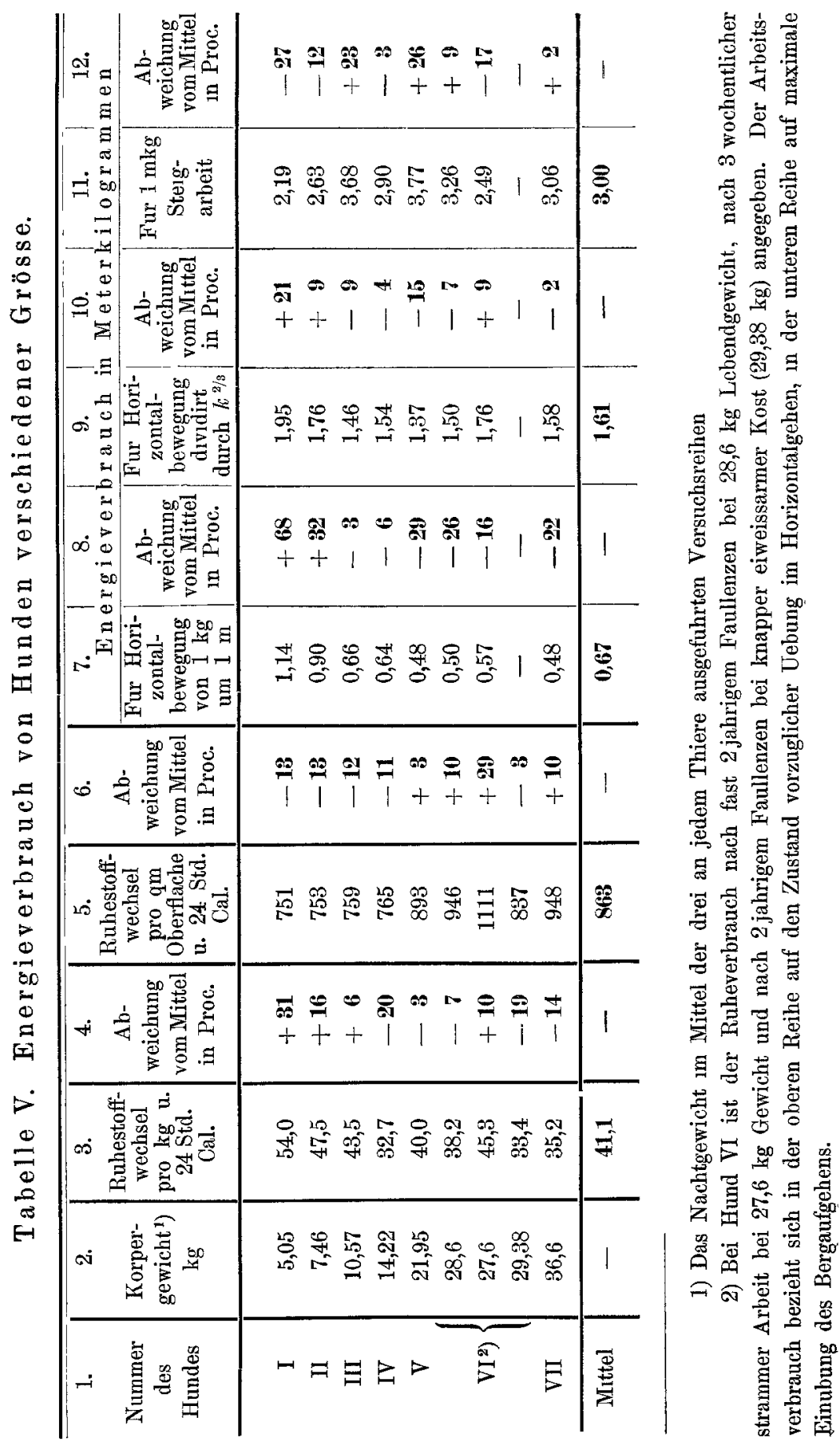


Einfluss der Geschwindigkeit, der Korpertemperatur u. der Uebung etc. 203

Bei Menschen und Pferden haben wir im Mittel fast genau den gleichen Stoffverbrauch fur das Meterkilogramm Steigarbeit gefunden wie beim Hunde, nämlich 2,96 bezw. $2,91 \mathrm{mkg}$.

Auch hier sind die individuellen Abweichungen nicht unerheblich. Von 8 gesunden Menschen brauchte der ökonomischste Arbeiter $2,73 \mathrm{mkg}$ gegen 3,56 als Hochstwerth; dies letztere allerdings bei schon unbequem steilem Anstieg; bei Pferden wurden unter dem Einflusse von leichten Anomalien der Sehnen und Gelenke noch erheblichere Abweichungen vom Durchschnitt beobachtet (siehe $\mathrm{Zunt} Z$ und Hagemann, Stoffwechsel des Pferdes, Tabelle LXXII S. 351). Fủr die Beurtheilung der Abweichungen muss man noch bedenken, dass der Verbrauch für die Steigarbeit durch Subtraction des Werthes fur den horizontalen Marsch vom Verbrauch beim Bergaufsteigen gefunden wird. Ein Thier, das wenig geschickt in der Ebene geht, aber beim Bergaufsteigen seine Muskeln zweckmässiger innervirt, wird uns daher zu kleine Werthe für das Meterkilogramm Steigarbeit ergeben. Die Zahlen der Tabelle $V$ bestätigen diese Ueberlegung. Bei Hund I und II sowie bei VI im Zustande der Uebung furs Bergaufgehen sehen wir in Col. 11 die Zahlen erheblich unter dem Mittel, in Col. 9 über demselben. Die umgekehrte Relation zeigen Hund III, V und VII sowie VI bei vorwiegender Uebung des horizontalen Ganges. -

Beim horizontalen Gang ergibt die Annahme, dass der Verbrauch nicht dem Gewichte $(\boldsymbol{K})$ sondern dem Werthe $\boldsymbol{K}^{2 / 3}$ proportional gehe, recht befriedigende Annaherung der Einzelwerthe an das Mittel fúr alle Versuchsthiere. Diese Annäherung ist hier sogar noch grösser als für den Ruhestoffwechsel (Col. 6). Das dürfte kein Zufall sein. v. Hoesslin, Archiv f. (Anat. u.) Physiol. 1888 S. 340 ff. hat durch eine eingebendere Analyse der Mechanik des Gehens dargethan, dass bei gleicher Geschwindigkeit der Kraftaufwand dem Körperquerschnitt, also $K^{2 / 3}$, proportional sein muss. - Dies geht am einfachsten aus folgender Erwägung hervor. Beim ersten Schritt muss, um dem ruhenden Körper die Gehgeschwindigkeit zu ertheilen, eine Arbeit $=1 / 2 \mathrm{~m} v^{2}$ geleistet werden.

Diese Arbeit ist aber, bei gleicher Geschwindigkeit $(v)$, der Masse, also dem Gewicht $K$, proportional. Von der erlangten Geschwindigkeit geht während des Schrittes ein Theil durch Reibung, Luftwiderstand, Hebung von Körpertheilen verloren. Dieser Bruchtheil, 
welcher bei ähnlich gebauten Thieren gleich sein dürfte, so lange die Geschwindigkeit dieselbe bleibt, muss also auch dem Gewichte $K$ proportional sein. Die zu seiner Compensirung nöthige Arbeit ist bei jedem Schritte aufs Neue aufzuwenden. Die Zahl der Schritte ist aber der Beinlänge und diese der dritten Wurzel des Körpergewichts umgekehrt proportional. Für die Wegeeinheit entspricht also die aufzuwendende Arbeit dem Ausdruck $\frac{K}{K^{1 / 3}}=K^{2 / 3}$. Selbstverständlich wird diese Proportion nur annähernd richtig sein; schon das wechselnde Verhältniss der Beinlängen zur Körpermasse muss Unterschiede bedingen, ebenso die Lagerung der Hauptmasse der Muskeln höher oder tiefer am Bein, die Beweglichkeit des Schulterblattes u. A. Dem entsprechend finden wir auch den auf gleiche relative Oberfläche bezogenen Verbrauch bei der gleichen Thierspecies, aber verschiedener Körpergrösse, nur in ziemlich engen Grenzen, bei versehiedenen Thierarten erheblicher schwanken. Dem Mittelwerthe 1,61 mkg beim Hunde entspricht ein Maximum von 1,95 bei Hund I, ein Minimum von 1,37 bei Hund V. Dagegen beträgt der correspondirende Mittelwerth beim Pferde 1,06; beim Menschen liegen die individuellen Schwankungen zwisehen 0,86 und $1,27 \mathrm{mkg}^{1}$ ). Die namentlich von Rubner vertretene Auffassung, dass der Ruhestoffwechsel desshalb der Körperoberfläche parallel gehe, weil er sich dem Wärmeverlnst durch die Haut anpasse, wird, wie fruher schon ausgefuhrt, den Thatsachen nicht gerecht. Rubner hat zwar in seiner jüngsten Veröffentlichung ${ }^{2}$ ) eine Reihe neuer Gesichtspunkte für die entscheidende Bedeutung der Temperaturverhältnisse bei der Regelung des Stoffwechsels angeführt, aber auch sie können nicht ganz befriedigen.

Rubner sagt mit Recht, dass die Beeinflussung des Stoffwechsels durch die Wärmeregulation nicht mit der Deckung des Verlustes erschöpft sei; es müsse sich der Stoffwechsel auch den Möglichkeiten der durch die Oberfläche vermittelten Entwärmung anpassen; hier-

1) Ich habe fruher schon darauf aufmerksam gemacht (dieses Archiv Bd. 68 S. 210), dass der Hund wahrseheinlich desshalb mehr Stoff beim Gehen verbraucht, als seiner Korpergrösse entspricht, weil er auch für das Stehen einen erheblichen Aufwand an Muskelkraft machen muss.

2) Die Gesetze des Energieverbrauchs bei der Eirnährung. Leipzig und Wien 1902. 
Einfluss der Geschwindigkeit, der Korpertemperatur u der Uebung etc. 205

durch sei die obere Grenze des Energieumsatzes begrenzt, diese aber bedinge ihrerseits wieder die Höhe des normalen Ruheumsatzes; dieser Gesichtspunkt der Entwärmung komme auch bei Kaltblütern in Betracht und bedinge hier die Anpassung des Umsatzes an die Korperoberflacche, welche mehrfach nachgewiesen worden ist. -

Demgegenuber ist denn doch zu betonen, dass die in unseren Gewässern lebenden Fische, bei welchen $\mathrm{Kn}$ a uthe die Gultigkeit der Oberfächenregel nachgewiesen hat, kaum je in die Gefahr der Ueberwärmung durch Muskelthatigkeit kommen, dass ferner bei Fischen die Ausgleichung zwischen Körper- und Umgebungstemperatur wohl in erster Linie durch die Kiemen vermittelt wird.

Beim Warmbliter begrenzt auch die Gefahr der Ueberwärmung nur ausnahmsweise die Grösse des Muskelstoffwechsels. Fur den Mensch hat Rubner selbst hervorgehoben, dass er normaler Weise durch Kleidung u. dergl. dafur sorgt, dass die chemische Warmeregulation bei ihm nicht in Anspruch genommen wird, und dass er auch der Entwärmung auf gleiche Weise zu Hulfe kommt, wenn er bei hoher Temperatur arbeiten muss. - Wir sehen also uberall, dass die Ableitung der Proportionalität zwischen Ruhestoffwechsel und $K^{2 / 3}$ aus der Wärmeregulation nicht durchfuhrbar ist. -

Da wir nun gesehen haben, dass der Verbrauch bei der hauptsächlichsten Arbeit der Thiere, dem horizontalen Gang, sehr annähernd proportional $K^{2 / 3}$ geht, da ferner die Annahme, dass ein gesetzmassiges Verhältniss zwischen dem Ruhestoffwechsel der Muskeln und ihrem Durchschnittsverbrauch bei Arbeit bestehe, sehr plausibel ist, liegt es nahe, in dem der Oberflache parallelen Wachsen des Verbrauchs fur die Locomotion die wesentlichste Ursache des gleichen Ganges des Ruhestoffwechsels zu suchen. Bei v. Hoesslin wie bei Rubner finden wir Beläge fur die Relation zwischen der Höhe des Arbeits- und des Ruhestoffwechsels. Ich kann im gleichen Sinne die Erfahrungen von Schumburg und mir an marschierenden Soldaten anführen. Im Laufe der Märsche, also wohl in Folge der ständigen stärkeren Muskelarbeit, stieg der Ruhestoffwechsel der jungen Männer, wảhrend gleichzeitig ihr Körpergewicht abnahm. Hier kann man immerhin annehmen, dass unter Fettschwund die Muskelmasse zugenommen habe, und dass die grössere Arbeit nur indirect, dadurch, dass sie das Wachsen der Muskeln anregte, den Ruhestoffwechsel gesteigert habe. Solche Annahmen reichen aber nicht mehr aus, wenn wir bei Hund VI 
sehen, dass sein Ruhestoffwechsel nach vierwöchiger Arbeit von 837 auf 1111 Calorien gestiegen ist, also um $33 \%$, wäbrend gleichzeitig sein Körpergewicht von 29,38 auf $27,6 \mathrm{~kg}$ gefallen war ${ }^{1}$ ). Hier ist eine der Zunahme des Ruheverbrauchs auch nur einigermaassen entsprechende Zunahme der Muskulatur undenkbar. Wir haben hier einen directen Beweis dafür, dass bei andauernd stärkerer Arbeit der Ruhestoffwechsel zunimmt, dass also die Qualitat der Muskeln sich derart ändern muss, dass der Ruheverbrauch grösser wird. Eine ahnliche qualitative Aenderung, welche zur Steigerung des Stoffwechsels in der Ruhe fuhrt, scheint bei sehr reichlicher Zufuhr von Eiweiss, derart, dass dieses allein zur Deckung des Stoffbedarfs ausreicht, sich zu entwickeln. Pflug er fand bei seinen Versuchen an Katzen, dass die Steigerung des Stoffwechsels der Zunahme des Eiweissbestandes des Körpers proportional erfolgte; das ist aber keine allgemeine Regel: die Versuche von Frentzel und Schreuer ${ }^{2}$ ) ergaben eine die Zunahme des Stickstoffbestandes am Körper sehr erheblich ubertreffende Zunahme der Oxydationsprocesse.

Die Labilität der activen Gewebe des Kỏrpers, ihre Zersetzlichkeit im Ruhezustande ist also eine nicht unerheblich wechselnde. Sie nimmt zu in Folge stärkerer Beanspruchung und ist desshalb bei kleineren Thieren, welche fur ibre Fortbewegung relativ mehr arbeiten

1) Man kann gegen diese Berechnung emwenden, dass der Hund bei den Ruheversuchen am 14. October 1893 nicht ganz nuchtern war. Er hatte vor sechs Stunden verzehrt:

$42 \mathrm{~g}$ Re1s, $17 \mathrm{~g}$ Fleischmehl und $7 \mathrm{~g}$ Fett,

eine Stunde darauf noch $150 \mathrm{~g}$ Rohrzucker. Die Resorption des Rohrzuckers verlauft ziemlich rasch, schneller jedenfalls als die einer aquivalenten Starkemenge. Wir uberschatzen daher sicher die bei diesem Versuche mitspielende Verdauungsarbeit erheblich, wenn wir sie so hoch veranschlagen, wie sie Magnus Levy an dem gleichen Thier nach Aufnahme von $300 \mathrm{~g}$ Reis gefunden hat (dieses Archiv Bd. 55 S. 54 Tab. XXI). Hier betrug die Steigerung des Sauerstoffverbrauchs im Mittel von drei Versuchen $19,6 \mathrm{ccm}$ pro Minute. Ziehen wir diese Zahl vom Ruheverbrauch $(180,4 \mathrm{ccm} \mathrm{0)} \mathrm{ab,} \mathrm{so} \mathrm{bleibt} \mathrm{als} \mathrm{Ruheverbrauch}$ $160,8 \mathrm{ccm} 0$ bei dem Resp.-Quot. 0,867. Hieraus berechnet sich unter der Annahme, dass der Zufuhr entsprechend $10 \mathrm{mg} \mathrm{N}$ pro Minute umgesetzt wurden, ein Kraftumsatz $=40.3$ Cal. pro Kilogramm und 24 Stunden bezw. 988 Cal. pro Quadratmeter Oberflache und 24 Stunden. Mit dieser sicher zu grossen Correctur ist der Verbrauch des trainirten Thieres immer noch um 18\% hoher als zur Zelt anhaltender Unthatigkeit.

2) Frentzel und Schreuer, Der Nutzwerth des Fleisches. Engelmann's Archiv 1902 S. 282. 
mússen als grosse, in gleichem Maasse gesteigert wie die Locomotionsarbeit, d. h. im Verhältniss des Quadrats der dritten Wurzel aus den Gewichten.

Mit der Annahme, dass die Beziehung zwischen $K^{2 / 3}$ und Horizontalbewegung die primäre, die zum Ruhestoffwechsel die secundäre ist, stimmt auch die geringere Abweichung des Stoffverbrauchs im Gehen von dem berechneten Mittelwerth. Die Extreme sind furr den Verbrauch

$$
\begin{aligned}
& \text { im Gehen }+21 \text { und }-15 \% \text {, } \\
& \text { in Rube }+29 \text { und }-13 \% \text {. }
\end{aligned}
$$

Wenn die Mechanik des Gehens, speciell die Grösse des einzelnen Schrittes fur den Stoffaufwand entscheidend ist, muss, wenn wir den Verbrauch pro Kilogramm und Meter mit der Beinlänge, statt mit $K^{1 / 3}$ multipliciren, auch ein gleicher Werth fur die Thiere verschiedener Grösse herauskommen. Leider habe ich die Messung der Beinlängen bei meinen 2 Versuchsthieren versäumt, und auch Dr. Slowtz off hat sie nur in 4 Fällen ausgefuhrt. Fur diese 4 Fälle stelle ich die Zahlen in Tab. VI zusammen. Es wurde das Mittel der Länge von Vorder- und Hinterbeinen genommen und damit der Verbrauch pro Kilogramm und Meter multiplicirt.

Tabelle VI.

\begin{tabular}{c|c|c|c|c|c}
\hline \hline $\begin{array}{c}\text { Nummer } \\
\text { des } \\
\text { Hundes }\end{array}$ & $\begin{array}{c}\text { Korper- } \\
\text { gewicht }\end{array}$ & $\begin{array}{c}\text { Beinlange } \\
\text { (Mittel) } \\
\mathrm{cm}\end{array}$ & $\begin{array}{c}\text { Verbrauch fur hornzont. Bewegung } \\
\text { in Meterkilogramm }\end{array}$ \\
\cline { 4 - 6 } & $\begin{array}{c}\text { pro Kilo } \\
\text { gramm und } \\
\text { Meter Weg }\end{array}$ & $\begin{array}{c}\text { pro Kilogramm und Meter } \\
\text { Weg multiplicirt mit }\end{array}$ \\
\hline II & 5,05 & 32,5 & 1,14 & 37 & $K^{1 / 3}$ \\
III & 10,57 & 36,7 & 0,66 & 24 & 1,95 \\
IV & 14,22 & 52 & 0,64 & 33 & 1,46 \\
VII & 36,6 & 74 & 0,48 & 35 & 1,58
\end{tabular}

Ausser bei der schwangeren Hündin III weichen die Ziffern der vorletzten Columne nur wenig von einander ab.

Recapitulirend sei zum Schlusse noch hervorgehoben:

1. Die Geschwindigkeit beeinflusst im Gegensatz zu Menseh und Pferd bei Hunden nicht sichtlich die Grössedes Stoffverbrauchs für die Wegeinheit.

2. Bei erhöhter Kórpertemperatur ist der Stoffverbrauch des ruhenden Körpers und der für die Athemarbeit erhöht; die Muskeln leisten aber einebe- 
stimmte Arbeit mit demselben Stoffaufwand wie bei normaler Temperatur.

3. Die Grösse des Verbrauchs für die einzelnen Leistungen der Muskulatur wird durch Uebung sehr erheblich herabgesetzt; die Uebung für eine bestimmte Arbeit bedingt aber keine Ersparniss bei andern nichtspeciell geübten Arbeiten; mit der grósseren Uebung der Muskulatur geht eine Steigerung des Ruhestoffwechsels einher, ebenso wie mit überschussiger Eiweissernahrung. 